\title{
Epithelial-Mesenchymal Transition: The Cancer Connection
}

\section{Homer S Black}

Department of Dermatology, Baylor College of Medicine, Houston, Texas 77030, USA

*Corresponding author: Homer S Black, Department of Dermatology, Baylor College of Medicine, Houston, Texas 77030, USA, Tel: 832-741-1052; E-mail: hblack@bcm.tmc.edu

Received date: Apr 20, 2015; Accepted date: Apr 21, 2015; Published date: Apr 28, 2015

Copyright: $\odot$ 2015, Homer S Black. This is an open-access article distributed under the terms of the Creative Commons Attribution License, which permits unrestricted use, distribution, and reproduction in any medium, provided the original author and source are credited.

\section{Editorial}

Epithelial-to-mesenchymal cell Transition (EMT) was first recognized as a fundamental event in embryogenesis. EMT, and its reverse process, Mesenchymal-Epithelial Transition (MET), are critical to morphogenesis and are involved in the development of many tissues and organs and events occurring in the developing embryo [1]. EMT results in profound morphological and phenotypic changes to a cell [2]. In this process, epithelial cells disassemble cell-cell junctions [3]; there is reorganization of the cytoskeleton [4]; increased cell motility [1]; and invasion occurs, (requisite for metastasis) [5] - all hallmarks of cancer. EMT has been categorized into three types. Type I is developmental; Type II involves fibrosis and wound healing; and Type III is involved in cancer [6]. It is Type III that we are concerned with here.

An excellent review of regulatory mechanisms that mediate MET in cancer appears in the current issue of the Journal (Boulding et al., J Integr Oncol 4.1, 2015). Therein the authors discuss the multi-layered epigenetic regulation of gene transcription during EMT that results in cancer. The overarching regulatory mechanism of gene transcription involves changes in chromatin structure, i.e., heterochromatin versus euchromatin. This involves 1) post-translational modifications (PTMs) of histone proteins; 2) DNA methylation; 3) ATP-dependent chromatin remodeling complexes; 4) exchange of standard histones with variants; and 5) the action of non-coding RNAs. The first, PTMs of the amino tails of histones is a particularly important modulator of gene expression. The PTM is accomplished by changing chromatin structure or tethering of transcriptional regulators to the epigenetic template. These changes are accomplished by numerous histonemodifying enzymes (HDACs) that may involve acetylation and deacetylation. Histone acetylation, particularly of lysine residues, neutralizes their basic charge, thus destabilizing higher order chromatin-folding and consequently allowing transcription. On the other hand, de-acetylation is catalyzed by histone deacetylases and represses transcription. Over expression of HDACs is associated with increased proliferation, differentiation, invasion, and metastasis and has been shown to occur in several types of cancer including breast, gastric, colorectal, prostrate, and kidney.

Activation and repression of transcription can result from histone methylation. Depending upon the specific amino acid residues, mono-, di-, and tri-methylations may occur. Methylation increases the hydrophobicity of histone side-chains that will increase the affinity for transcriptional activators or repressors - depending on the modified amino acid residue. Likewise, de-methylation is catalyzed by several lysine methyl transferases that interact with EMT-TFs to initiate EMT and is associated with increasing invasiveness of breast cancer. Generally, demethylase activity is a hallmark of cancer regulation and lysine specific demethylase-1 overexpression is found in a number of types of cancer, e.g., bladder, lung, ovarian, and prostate. It should be noted that not only modifications of histone play a role in gene expression, but it is well known that direct methylation of DNA also plays an important role in transcriptional silencing.

Also, it is known that signal transduction pathways play an important role in relaying information from the cell surface to the nucleus that, in turn, modulates gene expression. Signal transduction kinases are believed to shuttle between the cytoplasm and nucleus in resting cells to facilitate basal NF- $\mathrm{kB}$ transcriptional activity. A number of NF-kB family members have been shown to be involved in MET initiation and regulation of cells that have undergone a MET program. Nevertheless, epigenetic control is highly complex and involves regulation of several markers that can be used to identify the stages of MET. Epithelial markers include E-cadherin, Catenins, and Claudins. Mesenchymal markers include N-cadherin, Laminin, Fibronectin, and Vimentin. These markers define the phenotype of the cell. The loss of E-cadherin, an adherens junction protein and a key repressor of cellular metastasis, initiates EMT and enhances tumor metastasis.

MicroRNAs (miRNAs) are short non-coding RNA molecules that selectively bind to target messenger RNAs (mRNA) that posttranscriptionally regulate mRNA expression. Some miRNAs have been linked to EMT regulation.

This well referenced (158 citations) review shows how complex and intricate the multi-layered epigenetic regulatory mechanisms are that mediate EMT and how changes in the epigenetic landscape can lead to cancer. The review ends with a section on epigenetic therapy as a path to an effective treatment of cancer. As many of the epigenetic aberrations in the global landscape are reversible, inhibitors of histone modifying enzymes have shown promise as cancer therapeutics and some have already been approved for clinical use. EMT generates a subpopulation of cancer or cancer-like stem cells (CSCs) that are capable of extensive proliferation and differentiation. These cells are stem cells within a tumor and are capable of self- renewal and maintaining their initiating capacity [7]. These stem cells have the ability to cause tumor recurrence after therapy and thus new EMTbased epigenetic therapies must also focus on this important subpopulation of cells that give rise to metastasis and cancer recurrence.

\section{References}

1. Thiery JP, Acloque H, Huang RY, Nieto MA (2009) Epithelialmesenchymal transitions in development and disease. Cell 139: 871-890.

2. Thiery JP, Sleeman JP (2006) Complex networks orchestrate epithelialmesenchymal transitions. Nat Rev Mol Cell Biol 7: 131-142.

3. Schmalhofer O, Brabletz S, Brabletz T (2009) E-cadherin, beta-catenin, and ZEB1 in malignant progression of cancer. Cancer Metastasis Rev 28: 151-156. 
Citation: Black HS (2015) Epithelial-Mesenchymal Transition: The Cancer Connection. J Integr Oncol 4: e108. doi:10.4172/ 2329-6771.1000e108

Page 2 of 2

4. Berx G, Raspe E, Christofori, G, Thiery JP, Sleeman JP (2007) Pre-EMTing metastasis? Recapitulation of morphogenetic process in cancer. Clin Exp Metastasis 24: 587-597.

5. Brabletz T, Jung A, Reu S, Porzner M, Hlubek F, et al. (2001) Variable betacatenin expression in colorectal cancers indicates tumor progression driven by the tumor environment. Proc Natl Acad Sci USA 98: 10356-10361.
6. Kalluri R, Weinberg RA (2009) The basics of epithelial-mesenchymal transition. J Clin Invest 119: 1420-1428.

7. Kong D, Li Y, Wang Z, Sarkar FH (2011) Cancer stem cells and epithelialto-mesenchymal transition (EMT)-phenotypic cells: Are they cousins or twins? Cancers 3: 716-729. 